\title{
Dynamic Carpooling: for Better Environment
}

\author{
${ }^{1}$ S. Naga Jyothi, ${ }^{2}$ T. Haveela, ${ }^{3}$ T. Geeshpa, ${ }^{4}$ R. Swapna, ${ }^{5}$ T.Sumallika \\ ${ }^{1,2,3,4}$ IV year Students, ${ }^{5}$ Assistant Professor, IT Dept. , Gudlavalleru Engineering College, India.
}

Abstract - One of the main reason for increasing the pollution is rapid growth in vehicles .So as to reduce the number of vehicles we proposed an application named "Dynamic Carpooling Application for better environment".

Carpooling is the sharing of car journey so that more than one person travels in a car and prevents the need for others to have to drive to a location themselves. It is an application of finding car in which drivers who are travelling to work alone can ask other passenger to share the ride. By this we can reduce the vehicles and pollution. The major functioning of this project includes, the person enters his destination and he receives nearby ride request. So that, this person sends a message that he will be coming to the share ride. And they will share a ride.

Keywords: Carpooling, share ride, integration, autonomous cars.

\section{INTRODUCTION}

As we know that the pollution is increasing day by day we need to take some measures to prevent it. For example, in India according to a WHO Survey of 1650 world cities is the worst of any major city in the world. It also effects the districts around Delhi. Air pollution in India is estimated to kill about 2.5 million people every year. One of the main reason for increasing the pollution is rapid growth in vehicles .So as to reduce the number of vehicles we proposed an application named "Dynamic Carpooling Application for better environment".

Carpooling is the sharing of car journey so that more than one person travels in a car and prevents the need for others to have to drive to a location themselves. It is an application of finding car in which drivers who are travelling to work alone can ask other passenger to share the ride. By this we can reduce the vehicles and pollution. The major functioning of this project includes, the person enters his destination and he receives nearby ride request. So that, this person sends a message that he will be coming to the share ride. And they will share a ride.

\subsection{Problem Statement}

In our country there are many applications for carpooling. But the usage of that applications is very less and it is limited to some places only. So we proposed a system called Carpooling Application for Better Environment.

\subsection{Scope of the Project}

Email scheduling makes your entire outbox more flexible. Scheduling allows you to always acknowledge your coworker's free time and avoid sending non-urgent messages during evenings and weekends. Whether they check their email during these times or not, they will likely be more receptive to emails received during business hours, and you get the benefit of being able to click send anytime that's convenient for you.

\section{SySTEM ANALYSIS}

System Analysis is the process of analyzing a system with the potential goal of improving or modifying the system. Analysis is breaking down the problem into smaller elements for study and ultimately providing better solution. During the process of system development analysis is an important aspect. This involves gathering and interpreting facts, diagnosing the problem and using the information to recommended improvements to the system. Ultimately, the goal is to give a computerized solution.

\section{1 existing system}

There are many existing applications for carpooling like Bla Bla car, Pool My Ride etc. But in our country the usage is limited to some metropolitan cities only. So we proposed a system called Carpooling for Better Environment

\subsection{Limitations of Existing System}

1. You won't have much freedom or flexibility with your schedule and activities. Because there are several people traveling together, you usually have to leave at the same time. ...

2. You won't have much privacy. ...

3. The designated driver is responsible for his passengers.

\subsection{Proposed System}

The concept in the proposed system is people of different places can share their ride with other people. So we can reduce traffic and reduce usage of fuel and it is also pocket friendly.

\subsection{Advantages of Proposed System:}

1. Reduce travel cost. 
2. Reduce Fuel cost.

3. Reduce stress of driving.

4. Reduce the number of vehicles and Pollution.

5. Autonomous Cars.

\subsection{Literature Survey}

By verifying the websites like Pool My Ride and BlaBla car we came to know that what is Carpooling and demerits of Carpooling, then we have decided to do this project for a better environment and for safety purpose.

\subsection{Feasibility Study}

A feasibility study is a preliminary study undertaken to determine and document a project's viability. The term feasibility study is also used to refer to the resulting document. These results of this study are used to make a decision whether to proceed with the project. It is an analysis of possible alternative solutions. It, for example, can decide whether an order processing be carried out by a new system more efficiently than the previous one.

\section{Economic Feasibility}

Economic analysis is most frequently used for evaluation of the effectiveness of the system. More commonly known as cost/benefit analysis the procedure is to determine the benefit and saving that are expected from a system and compare them with costs, decisions is made to design and implement the system. This is an important input to the management because very often the top management does not like to get confounded by the various technicalities that bound to be associated with a project of this kind.

\section{Technical Feasibility}

Technical feasibility centers on the existing manual system of the test management process and to what extent it can support the system. According to feasibility analysis procedure the technical feasibility of the system is analyzed and the technical requirements such as software facilities, procedure, inputs are identified. It is also one of the important phases of the system development activities.

\section{Behavioral Feasibility}

People are inherently resistant to change and computer has been known to facilitate changes. An estimate should be made of how strong the user is likely to move towards the development of computerized system.

\subsection{System Requirement Specifications}

Software requirement specification is the basic step to develop a software development procedure. The SRS mean that translating the client ideas into a formal document. Before document, before moving in depth into design. Thus the output of the phase is a set of formally specified requirements, which hopefully are complete and consistence while input has none of these properties. The requirement specification phase terminates with production of the validation software requirement specification document.

\section{SOFTWARE REQUIREMENTS (minimum)}

1. Operating System -Windows 7 or latest version

2. Programming Language - HTML, Php

3. Software -Php

4. Application Server -Xamp Server

5. Database -MYSQL

\section{HARDWARE REQUIREMENTS (minimum)}

- Processor : Intel Core i5

- RAM : 4 GB

- Hard Disk : 1 TB

\section{SYSTEM DESIGN}

Once software requirements have been analyzed and specified, software design as the first of three technical activities design, code generation and test-that are require to build and software. Various stages in design are

$$
\begin{aligned}
& \text { Conceptual design. } \\
& \text { Database design. } \\
& \text { Functional design. }
\end{aligned}
$$

\section{Conceptual Design:}

The conceptual structure of a database is called schema. Schema shows the kind of data that exists in a database and how these are logically related to each other. A schema can be regarded as a blueprint that portrays, both, kind of data used in building a database and logical relationship, and must correctly represent their inter relationships. Schema is frequently depicted pictorially via, Data Flow diagram etc.

A database management system (DBMS) is computer software designed for the purpose of managing databases, a large set of structured data, and run operations on the data requested by numerous users. Typical examples of DBMSs include Oracle, DB2, Microsoft Access, Microsoft SQL Server, Firebird, PostgreSQL, MySQL, SQLite, FileMaker and Sybase Adaptive Server Enterprise. DBMSs are typically used by Database administrators in the creation of Database systems. Typical examples of DBMS use include accounting, human resources and customer support systems.

Originally found only in large companies with the computer hardware needed to support large data sets, DBMSs have more recently emerged as a fairly standard part of any company back office. 


\subsection{Database Design:}

\section{Description}

A DBMS is a complex set of software programs that controls the organization, storage, management, and retrieval of data in a database.

A DBMS includes: A modeling language to define the schema of each database hosted in the DBMS, according to the DBMS data model.

The four most common types of organizations are the hierarchical, network, relational and object models. Inverted lists and other methods are also used. A given database management system may provide one or more of the four models. The optimal structure depends on the natural organization of the application's data, and on the application's requirements (which include transaction rate (speed), reliability, maintainability, scalability, and cost).

The dominant model in use today is the ad hoc one embedded in SQL, despite the objections of purists who believe this model is a corruption of the relational model, since it violates several of its fundamental principles for the sake of practicality and performance. Many DBMSs also support the Open Database Connectivity API that supports a standard way for programmers to access the DBMS.

$\square$ Data structures (fields, records, files and objects) optimized to deal with very large amounts of data stored on a permanent data storage device (which implies relatively slow access compared to volatile main memory).

$\square \quad$ A database query language and report writer to allow users to interactively interrogate the database, analyze its data and update it according to the users privileges on data.

It also controls the security of the database.

Data security prevents unauthorized users from viewing or updating the database. Using passwords, users are allowed access to the entire database or subsets of it called sub schemas.

If the DBMS provides a way to interactively enter and update the database, as well as interrogate it, this capability allows for managing personal databases. However, it may not leave an audit trail of actions or provide the kinds of controls necessary in a multi-user organization. These controls are only available when a set of application programs are customized for each data entry and updating function.

A transaction mechanism, that ideally would guarantee the ACID properties, in order to ensure data integrity, despite concurrent user accesses (concurrency control), and faults (fault tolerance). $\square \quad$ It also maintains the integrity of the data in the database.

$\square \quad$ The DBMS can maintain the integrity of the database by not allowing more than one user to update the same record at the same time. The DBMS can help prevent duplicate records via unique index constraints; for example, no two customers with the same customer numbers (key fields) can be entered into the database. See ACID properties for more information (Redundancy avoidance).

$\square$ The DBMS accepts requests for data from the application program and instructs the operating system to transfer the appropriate data. When a DBMS is used, information systems can be changed much more easily as the organization's information requirements change. New categories of data can be added to the database without disruption to the existing system. Organizations may use one kind of DBMS for daily transaction processing and then move the detail onto another computer that uses another DBMS better suited for random inquiries and analysis. Overall systems design decisions are performed by data administrators and systems analysts. Detailed database design is performed by database administrators.

Database servers are specially designed computers that hold the actual databases and run only the DBMS and related software. Database servers are usually multiprocessor computers, with RAID disk arrays used for stable storage. Connected to one or more servers via a high-speed channel, hardware database accelerators are also used in large volume transaction processing environments.

\section{SYSTEM IMPLEMENTATION}

\subsection{Introduction}

Computer programming (often shortened to programming or coding) is the process of designing, writing, testing, debugging / troubleshooting, and maintaining the source code of computer programs. This source code is written in a programming language.

\subsection{Module Description}

The DYNAMIC CARPOOLING FOR BETTER ENVIRONMENT comprises the following major modules:

$\square$ ADMIN: The admin sends feedback to owner and stores the details about passenger.

$\square$ OWNER: Receives the feedback from admin and views the passenger's details.

$\square$ PASSENGER: Views the owner details and requests a ride. 


\section{System Architecture}

Carpooling is the sharing of car journey so that more than one person travels in a car and prevents the need for others to have to drive to a location themselves. It is an application of finding car in which drivers who are travelling to work alone can ask other passenger to share the ride. By this we can reduce the vehicles and pollution. The major functioning of this project includes, the person enters his destination and he receives nearby ride request. So that, this person sends a message that he will be coming to the share ride. And they will share a ride.

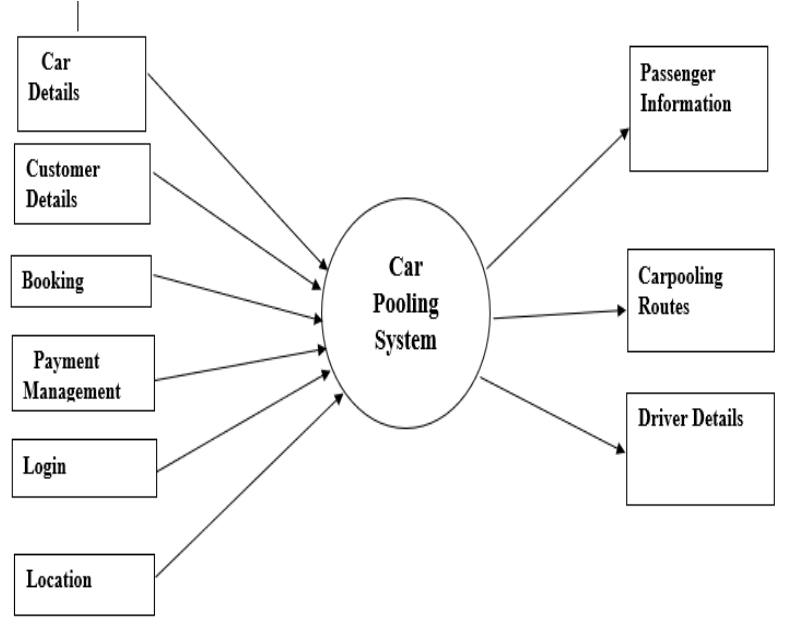

Fig 4.1 DATAFLOW DIAGRAM

\section{System TeSTING}

Testing is the process of detecting errors. Testing performs a very critical role for quality assurance and for ensuring the reliability of software. The results of testing are used later on during maintenance also. Testing is the process of trying to discover every conceivable fault or weakness in a work product.

Software testing is the process used to assess the quality of Computer Software. Software testing is an empirical technical investigation conducted to provide stakeholders with information about the quality of the product or service under test, with respect to the context in which it is intended to operate. This includes the process of executing a program or application with the intent of finding

Over its existence, computer software has continued to grow in complexity and size. Every software product has a target audience. For example, video game software has its audience completely different from banking software. Therefore, when an organization develops or otherwise invests in a software product, it presumably must assess whether the software product will be acceptable to its end users, its target audience, its purchasers, and other stakeholders. Software testing is the process of attempting to make this assessment.

Different levels of testing that have to be conducted are

\author{
Unit Testing \\ Integration Testing \\ System Testing \\ Implementation \& Maintenance Testing
}

\subsection{Unit Testing:}

Unit testing focuses verification effort on the smallest unit of software i.e. the module. Using the detailed design and process specifications testing is done to uncover errors within the boundary of the module. All the modules must be successful in the unit test before the start of the integration testing begins.

\subsection{Integration Testing:}

After the unit testing we have to perform integration testing. The goal here is to see if modules can be integrated properly, the emphasis being on testing interfaces between modules. This testing activity can be considered as testing the design and hence the emphasis on testing module interaction.

\subsection{System Testing:}

Here the entire software system is tested. The reference document for this process is the requirements document, and the goal of OS to see software meets its requirements.

\subsection{Implementation and Maintenance Testing:}

Maintenance testing is that testing which is performed to either identify equipment problems, diagnose equipment problems or to confirm that repair measures has been effective. It can be performed at either the system level, the equipment level, or the component level.

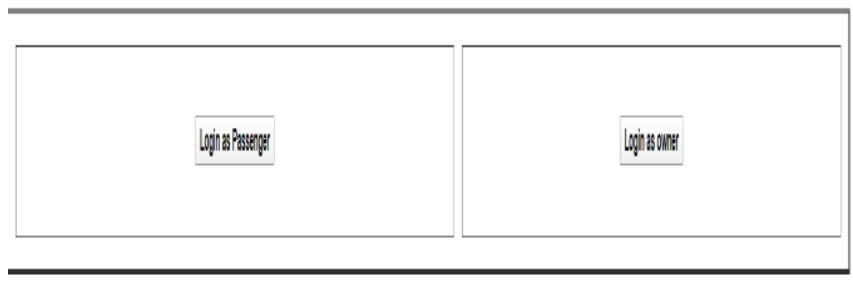




\begin{tabular}{|c|c|c|c|}
\hline HONE! & CHECH FOR CAR POOLING & $\triangle B O C T$ & $\underline{\text { LOGOCIT }}$ \\
\hline
\end{tabular}

OFFER RIDE!

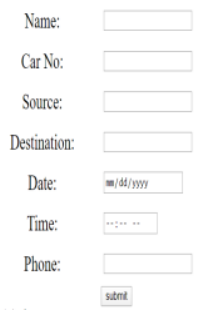

\begin{tabular}{|l|l||l|l|}
\hline \hline$\underline{\text { HONS }}$ & $\underline{\text { CHECH FOR CAR POOLING }}$ & $\underline{\text { ABOCI }}$ & LOGOCI \\
\hline
\end{tabular}

WELCOME TO OWNERS HOWE PAGE!

OWNER LOGIN GATEWAY!

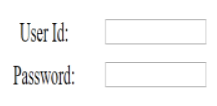

$\operatorname{login}$

Go To Main Merenl

Go To Passenger Login Gatemay?

Go Io Registration Page

$$
\text { Go I0 Mlain MeanI }
$$

Go To Owreer Login Gateray?

Go Io Registration Page

\begin{tabular}{|l|l|l|l|}
\hline \hline HONIL & CHECH FOR CAR POOLIG & HBOCI & LOGOCT \\
\hline
\end{tabular}

\section{PASSEGEOPS POOL!}

CHOOE MOLR POOLKG OPTOW

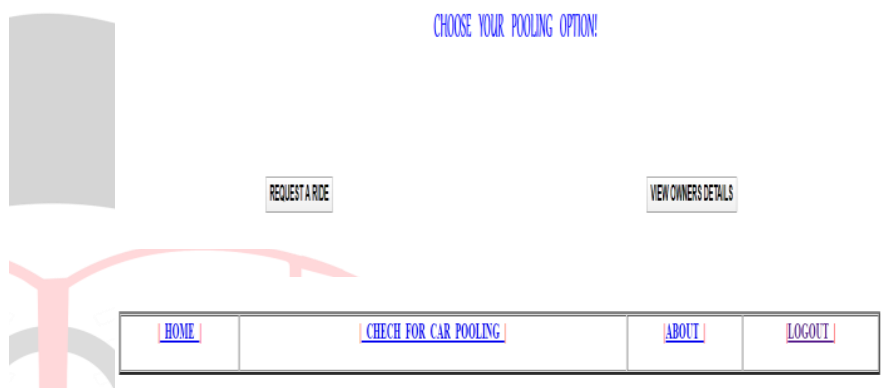

\section{OFFER RDE!}

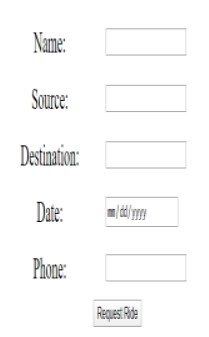

\section{Conclusion}

OFFRRE

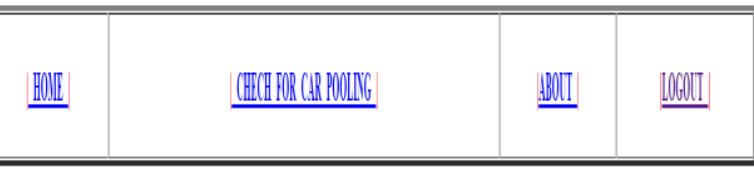

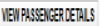

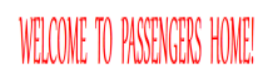

The "DYNAMIC CARPOOLING FOR BETTER ENVIRONMENT" is a web based application. In this application Manual work is replaced with a web based system. This web system acts as interface between admins and residents. If we follow this there is no scope of delays. Here we are creating a database to store the information about driver and passenger. This can be achieved by providing logins to them.

The technology used in this project are WAMP server, for designing and Php for connecting to the databases. By verifying the websites like Pool My Ride and BlaBla car we came to know that what is Carpooling and demerits of Carpooling, then we have decided to do this project for a better environment and for safety purpose 


\section{FUTURE ENHANCEMENTS}

- Make the application generalized, so that every village can use it to make the Adoption system easier.

- Mapping of Program outcomes can be implemented.

\section{REFERENCES}

[1] A carpooling recommendation system for taxicab is published by Zhang-Cited by 59 .

[2] Automated wireless carpooling system for an ecofriendly published by Megalingam -Cited by 20 .

[3] Real-time carpooling system-IEEE Conference Publication-IEEE Xplore by NV Pukhovskiy. Andriod based application for efficient carpooling with user IEEE Xplore.

[4] PK Binu. Briggs M., Future of Mobility: Introducing the New Business Models Frost \& Sullivan's Intelligent Mobility Workshop 05/07/2015

[5] Galizzi M.M., The Economics of Car-Pooling: A Survey for Europe, 2004

[6] Briggs M., Future of Mobility: Introducing the New Business Models Frost \& Sullivan's Intelligent Mobility Workshop 05/07/2015

[7] Galizzi M.M., The Economics of Car-Pooling: A Survey for Europe, 2004

[8] Baumert K, Herzog T, Pershing J (2005). Navigating the Numbers: Greenhouse Gas Data and International Climate Policy. World Resources Institute.

[9] Abu-Lebdeh G, Benekohal RF (2003). Design and evaluation of dynamic traffic management strategies for congested conditions. Transportation Research Part A: Policy and Practice, 37(2), 109-127.

[10] Lo HK, Chang E, Chan YC (2001). Dynamic network traffic control. Transportation Research Part A: Policy and Practice, 35(8), 721-744.

[11]Ritchie SG (1990). A knowledge-based decision support architecture for advanced traffic management. Transportation Research Part A: General, 24(1), 2737.

[12]Federal Highway Administration (2013). High Occupancy Vehicle Facilities. U.S. Department of Transportation. Retrieved from http://ops.fhwa.dot.gov/freewaymgmt/faq.htm\#f aq7

[13] Chan ND, Shaheen SA (2012). Ridesharing in North America: Past, Present, and Future. Transport Reviews, 32(1), 93-112.

[14] Ferguson E (1997a). The rise and fall of the American carpool: 1970-1990. Transportation, 24(4), 349-376.

[15] Park H, Gebeloff R (2011). Car-Pooling Declines as Driving Becomes Cheaper. New York Times. New York. 